OPEN ACCESS

Edited by:

Annalise Weckesser, Birmingham City University,

United Kingdom

Reviewed by:

Débora Godoy-Izquierdo

University of Granada, Spain

Bronwen Gillespie,

The University of Sheffield,

United Kingdom

*Correspondence:

Siladitya Bhattacharya

s.bhattacharya@abdn.ac.uk

Specialty section:

This article was submitted to Quality of Life,

a section of the journal

Frontiers in Global Women's Health

Received: 03 September 2021 Accepted: 29 November 2021 Published: 21 December 2021

Citation:

McPherson L and Bhattacharya S (2021) Measuring What Matters - $A$

Holistic Approach to Measuring Well-Being in Endometriosis. Front. Glob. Womens Health

2:770366.

doi: 10.3389/fgwh.2021.770366

\section{Measuring What Matters-A Holistic Approach to Measuring Well-Being in Endometriosis}

\author{
Lori McPherson ${ }^{1}$ and Siladitya Bhattacharya ${ }^{2 *}$ \\ ${ }^{1}$ Department of Obstetrics and Gynaecology, NHS Grampian, Aberdeen, United Kingdom, ${ }^{2}$ Department of Obstetrics and \\ Gynaecology, School of Medicine, Medical Sciences and Nutrition, University of Aberdeen, Aberdeen, United Kingdom
}

Endometriosis is a common condition which affects women in a number of ways and impairs their ability to live a full and meaningful life. Evaluative research has traditionally taken its cue from a medical approach which has forced women to choose one area of functioning as their primary concern, and tended to use a narrow definition of treatment success which ignores general well-being. While recent trials have included quality of life (QOL) measures as outcomes, these have not been able to capture the totality of the impact of the disease and its treatment on a woman's capability to do what she might want to do and be who she might want to be. A capability approach might overcome this barrier, but the available tools will need to be refined and validated in women with endometriosis before this can be integrated within everyday clinical and research practice.

Keywords: endometriosis, quality of life (QOL), capabilities, gynaecology, women

\section{INTRODUCTION}

One in ten women of reproductive age lives with endometriosis. The proliferation of hormone sensitive endometrial cells outside the uterus leads to cyclic bleeding, inflammation, and scarringmainly within the pelvis but also, occasionally, other parts of the body (1). The commonest symptoms of endometriosis are pelvic pain and infertility but the chronicity of the condition, side effects of treatment and the need for a surgical diagnosis can have a profound impact on many different aspects of a woman's life. The condition can progress over time and many women require multiple surgical procedures (2). The cornerstone of clinical management is symptom control and the conventional approach has been to dichotomise the physical symptoms of the disease into either pain or infertility before offering targeted treatment. Although many women present with both pain and infertility, they are often advised to prioritise one of these as hormonal treatments for pain can interfere with ovulation and are incompatible with attempts to conceive.

Randomised trials assessing the effectiveness of treatments for endometriosis have tended to respect this artificial division and focused on live-birth or severity of pain as outcomes. More recently, the importance of measuring QOL as a global indicator of treatment success has been recognised, but current methods for assessing QOL in endometriosis have been inconsistent and have not been designed to capture the totality of the impact of the condition on women's well-being $(3,4)$.

In this article we review outcomes reported in randomised trials of treatments for endometriosis and tools used to measure these. We also consider an alternative choice driven capability-based 
approach $(5,6)$. which aims to shift the focus from measuring utility (happiness) to evaluating functionings and capabilities in women (7).

\section{OUTCOMES AND TOOLS USED IN RANDOMISED CLINICAL TRIALS}

We undertook a computerised search of the Cochrane Library in order to identify reviews of randomised trials of treatments in endometriosis. From an initial list of 52 eligible articles, 3 were excluded as they (3) had been withdrawn from the Cochrane Library. Of the remaining 49, 29 were excluded after screening the abstracts as they did not conform to our inclusion criteria, leaving 20 articles for final analysis (Appendix 1). Fertility was the chosen primary outcome in 4 reviews, pain in 12 and both in 4 . In the reviews with pain as the primary outcome, fertility was considered a secondary outcome in 3; of the 4 reviews with fertility as a primary outcome, 1 review included pain as a secondary outcome.

Of the reviews focusing on fertility, 6 selected live birth rate as the outcome of interest (8-13). Hart et al. (14) reported clinical pregnancy rate and Brown and Farquhar (15) included all pregnancy outcomes; including live birth, miscarriage, and ectopic pregnancy etc. In actual fact, due to the nature of the data in the primary trials, only 3 of the Cochrane reviews were actually able to report live births, while the others reported rates of clinical pregnancy or viable pregnancy. In three reviews, all participants were undergoing fertility treatment (IVF or ICSI) $(8,10,11)$. Hughes et al. (9) included participants who were deemed infertile, having failed to conceive after 12 months of unprotected intercourse. While all 14 randomised control trials (RCTs) included in the systematic review by Balfort et al. (12) commented on fertility outcomes, only 3 included women with a diagnosis of infertility. Similarly, the review by Lu et al. (13) included 4 RCTs of which 3 focused on participants with a confirmed diagnosis of infertility. None of the 3 RCTs reviewed by Hart et al. (14) specifically included participants with infertility.

Pain was a key outcome for many of the reviews and the most common tool used to assess it was the visual analogue scale (VAS) which figured in 11 reviews (12-23). Eight reviews used dichotomous outcomes (e.g., improved or not improved) to assess pain $(14-16,18,19,21,23,24)$. The Biberoglu and Behrman rating scale was used by both Lu et al. (22) and Brown and Farquhar (15). This was modified to take into account the impact on work and loss of function by Abou-Setta et al. (17), who also used the Total Endometriosis Severity Profile (TESP) to assess pain. The Endometriosis Symptoms Severity Score (ESSS) was used by 2 reviews to assess pain $(15,23)$. Two reviews used a specific set of Chinese validated outcomes to assess pain $(15,25)$. Flower et al. (26) and Brown and Farquhar (15) assessed pain using the 15-point Guideline for Clinical Research on New Chinese Medicine for Treatment of Pelvic Endometriosis scale, while Brown et al. (20) used the verbally reported scale (VRS). One review used an unspecified "pain questionnaire" to assess pain (27).
Three reviews reported recurrence of disease, as assessed at repeat laparoscopy using the standard or revised American Fertility Society (AFS) $(18,19,24)$, revised American Society for Reproductive Medicine (ASRM) score or Endoscopic Endometriosis Classification (EEC) score (19) as their primary outcome.

Seven reviews also reported on adverse outcomes, complication rate or side effects of treatment $(10,11,15,16,21,22,24)$. Farquhar et al. (24) was the only review to select patient satisfaction with treatment. Although none of the reviews reported on QOL as a primary outcome, 7 aimed to assess this as a secondary outcome measure. Only 3 were able to report data on QOL. Fu et al. (21) and Zhu et al. (25) used the short form 36 (SF-36) as a QOL instrument. Balfort et al. (12) used the European Quality of life 5 dimension (EQ-5D) index summary, the short form 12 (SF-12) and the Endometriosis Health Profile-30 (EHP-30).

These data confirm the traditional emphasis on pain and fertility, rather than QOL as primary outcomes in those undertaking randomised trials in endometriosis as well as those engaged in evidence synthesis. Although some systematic reviews have included QOL as a secondary outcome, the variety of tools used (not all of which are disease-specific) make the results difficult to interpret. Yet, endometriosis is a multidimensional disease which does not just affect a woman physically but also causes anxiety and depression, compromises social relationships (28) and affects opportunities for education, employment and reproduction. QOL measures have sought to capture general well-being in women with endometriosis using a variety of techniques but, as yet, there is no consensus on the best way of achieving this (4). The most commonly used tools were SF-36 and the EHP-30. SF-36 is a generic QOL instrument which has been validated for use in endometriosis. Although it allows comparison between different diseases and the general population, it is likely to miss certain unique aspects of endometriosis such as dyspareunia, often described as a debilitating symptom. EHP-30 is a disease-specific tool for endometriosis, but while it examines 11 different domains, it does not provide a single score for QOL, thus limiting its appeal amongst researchers seeking a single outcome for a clinical study (29).

This review included studies conducted mainly in medium and high income countries with the findings written in English. As such, it may not be entirely representative of women across the world especially those from low income countries. This is a limitation of the review when considering its implications in a global context.

\section{A NEW CORE OUTCOME SET FOR CLINICAL TRIALS}

Recently, a multidisciplinary international group, including women living with endometriosis, has used robust methods to standardise outcome selection, collection and reporting across randomised trials and systematic reviews in endometriosis (4). Three core outcomes for trials evaluating potential treatments for pain and other symptoms associated with endometriosis 
include overall pain, improvement in the most troublesome symptom and QOL. In trials evaluating potential treatments for infertility associated with endometriosis, 8 core outcomes were identified. These include viable intrauterine pregnancy confirmed by ultrasound, pregnancy loss (including ectopic pregnancy, miscarriage, stillbirth, and termination of pregnancy), live birth, time to pregnancy leading to live birth, gestational age at delivery, birthweight, neonatal mortality, and major congenital abnormalities. Adverse events and patient satisfaction with treatment were identified as core outcomes applicable to all trials (4).

\section{ARE WE MEASURING WHAT MATTERS?}

The core outcome set includes QOL in women with pain, but not infertility associated with endometriosis. While endometriosis is associated with psychological morbidity and reduced QOL, mainly due to the impact of chronic pelvic pain (3), the experience of childlessness can affect well-being (30). A systematic review (3) has highlighted the paucity of studies using QOL as an outcome, along with a lack of long term follow up studies and methodological issues in the design of QOL instruments, especially as generic tools have limitations in terms of measuring chronic conditions which fluctuate over time. Despite their obvious advantages, some of the common diseasespecific questionnaires have been found to have design problems, and it has been suggested that it might be prudent to use more than one type of tool (3).

Standard QOL tools such as SF-36 and EQ-5D can provide an estimate of the effect of endometriosis on day-to-day functioning and provide health funders and policy makers with quality adjusted life years (QALY) for comparisons with other health conditions in order to inform health policy. Yet QALYs are hardly ever used to inform decision making at a personal level and patients can (and do) have the right to refuse treatments on grounds of personal autonomy. Thus, these generic QOL measures fall short of being able to describe the totality of a woman's experience, provide a comprehensive estimate of the full impact of an intervention on a woman's well-being or act as a practical guide to joint clinical decision making in everyday practice.

Disease-specific tools can capture the impact of endometriosis on different aspects of a woman's life but cannot fully incorporate a woman's inability to realize her reproductive and other life plans. This is problematic, as any health gain in a narrow physical domain could come at an unacceptably high cost in terms of unfulfilled expectations and absent opportunities. A woman who chooses not to have children might have the same fertility outcome as someone who is unable to have children either due to endometriosis or the effects of its treatment, but the two have very different states of well-being.

The capabilities approach developed by Amartya Sen (7) and Nussbaum (31) allows clinicians and researchers to think more globally in terms of measuring the impact of a condition and its treatment, not just on their immediate physical state but their ability to lead a life which has value. Capabilities represent functions which are essential to a flourishing life or "eudaimonia" (5), including attributes such as normal life span; bodily health; bodily integrity; senses; imagination and thought; emotions; practical reason; affiliation; the ability to appreciate and interact with other species; play and control over one's environment (32). Functionings are things a person can do (exercise, work, play) or achieve (employment, parenthood) (33) but measurement of achieved functionings may be insufficient for a complete assessment of well-being which should also include a person's freedom to achieve their goals in life (34). Thus, measuring achievement (ability to perform physical and mental tasks) is less meaningful unless considered alongside capabilities (34). This approach, which accepts the multidimensional nature of wellbeing and recognises the importance of choice, represents a more holistic method of reviewing health in endometriosis; which not only limits what women can do in terms of everyday tasks, but also what they might plan to achieve in their lives (35).

\section{EXISTING CAPABILITY MEASURES}

A number of research groups have attempted to develop capability-based measures which currently include tools such as the Oxford Capability instrument (OxCap) $(36,37)$, the Adult Social Care Outcome Toolkit (ASCOT) $(38,39)$ and the ICEpop CAPability measure (ICECAP) $(40,41)$. OxCap (Oxford Capability) comprises a 64 item questionnaire based on analysis of secondary data from household and panel surveys and has since been adapted to different settings $(37,42)$. ASCOT was developed to measure capability specifically in relation to social care and has since evolved through four versions, with Sen's capability theory appearing only relatively late in its development $(38,43)$. ICECAP $(40)$ was initially targeted at older people but subsequent work has been done to develop the five-attribute ICECAP-A measure for the adult population (41) and the seven-attribute ICECAPSCM, a supportive care measure for use with people at the end of life (44). These tools have varied in their treatment of the issue of capabilities vs. functionings as well as their basis for the capabilities evaluated. Whilst the identification of attributes for the OxCap has been expert led, the ICECAP and ASCOT instruments were developed through participatory methods involving qualitative work with representative groups. Nussbaum's concept of central capabilities (31) has influenced the approach used by Anand et al. (36), who used questions from the British Household Panel Survey to assess capability and refine the later versions of OxCap. In contrast, ASCOT and ICECAP started with the premise that it was important to venture beyond health, with the move to capability coming later. For ICECAP researchers, the impetus for a capability approach came from results from qualitative research (40).

All current approaches are still at a relatively early stage but there is increasing evidence of the use of these measures in research (45). Areas for further development have been identified; such as the use of different approaches to generate both measurement systems and their valuation, exploration of their sensitivity over the life course (46) and ways of using the 
capability approach in decision making. A fundamental problem is the fact that a measure of capability cannot be generated from questions about the ability to undertake actions (33). In addition, measuring an individual's entire capability set is challenging and cannot just be based on the totality of functionings (33). There have been some initial suggestions about how these issues can be addressed, but it is clear that much more work is needed.

\section{CONCLUSION}

Endometriosis is a common condition which affects women in a number of ways and impairs their ability to live a full and meaningful life. Evaluative research has traditionally taken its cue from a medical approach which has forced women to choose one area of functioning as their primary concern and tended to use a narrow definition of treatment success which ignores general well-being. While recent trials have included QOL measures as outcomes, these have not been able to capture the totality of the impact of the disease and its treatment on a woman's capability to

\section{REFERENCES}

1. NHS.uk. Conditions-Endometriosis. Available online at: https://www.nhs.uk/ conditions/endometriosis/ (accessed: Oct 28, 2021).

2. Saraswat L, Ayansina D, Cooper K, Bhattacharya S, Horne A, Bhattacharya S. Impact of endometriosis on risk of further gynaecological surgery and cancer: a national cohort study. BJOG. (2018) 125:64-72. doi: 10.1111/1471-0528.14793

3. Jones GL, Kennedy SH, Jenkinson C. Health-related quality of life measurement in women with common benign gynecologic conditions: a systematic review. Am J Obstet Gynecol. (2002) 187:501-11. doi: 10.1067/mob.2002.124940

4. Duffy J, Hirsch M, Vercoe M, Abbott J, Barker C, Collura B, et al. A core outcome set for future endometriosis research: an international consensus development study. BJOG. (2020) 127:967-74. doi: 10.1111/1471-0528.16157

5. Feminist Interpretations of Aristotle Edited by Cynthia A. Freeland. Available online at: https://www.psupress.org/books/titles/0-271-01729-5. html (accessed: Oct 28, 2021).

6. Walker M. Towards a capability-based theory of social justice for education policy-making. J Educ Policy. (2006) 21:16385. doi: 10.1080/02680930500500245

7. Amartya Sen. Capability and Well-Being-Oxford Scholarship. Available online at: https://oxford.universitypressscholarship.com/view/10.1093/0198287976. 001.0001/acprof-9780198287971-chapter-5 (accessed: Oct 28, 2021)

8. Sallam HN, Garcia-Velasco JA, Dias S, Arici A, Abou-Setta AM, Jaafar SH. Long-term pituitary down-regulation before in vitro fertilization (IVF) for women with endometriosis. Cochrane Database Syst Rev. (2006) 2006:CD004635. doi: 10.1002/14651858.CD004635

9. Hughes E, Brown J, Collins JJ, Farquhar C, Fedorkow DM, Vanderkerchove P. Ovulation suppression for endometriosis for women with subfertility. Cochrane Database Syst Rev. (2007) 2007:CD000155. doi: 10.1002/14651858.CD000155

10. Georgiou EX, Melo P, Baker PE, Sallam HN, Arici A, Garcia-Velasco JA, et al. Long-term GnRH agonist therapy before in vitro fertilisation (IVF) for improving fertility outcomes in women with endometriosis. Cochrane Database Syst Rev. (2019) 2019:CD013240. doi: 10.1002/14651858.CD0 13240

11. Benschop L, Farquhar C, Poel $\mathrm{N}$ van der, Heineman MJ. Interventions for women with endometrioma prior to assisted reproductive technology. Cochrane Database Syst Rev. (2010) CD008571. doi: 10.1002/14651858.CD008571 do what she might want to do and be who she might want to be. The multidisciplinary implementation of a capability approach might overcome this barrier, but the available tools will need to be refined and validated in women with endometriosis before this can be integrated within everyday clinical and research practice.

\section{AUTHOR CONTRIBUTIONS}

LM drafted the manuscript after conducting a review of the literature. SB edited the manuscript and expanded it. Both authors contributed to the article and approved the submitted version.

\section{SUPPLEMENTARY MATERIAL}

The Supplementary Material for this article can be found online at: https://www.frontiersin.org/articles/10.3389/fgwh. 2021.770366/full\#supplementary-material
12. Bafort C, Beebeejaun Y, Tomassetti C, Bosteels J, Duffy JM. Laparoscopic surgery for endometriosis. Cochrane Database Syst Rev. (2020) 10:CD011031. doi: 10.1002/14651858.CD011031

13. Lu D, Song H, Li Y, Clarke J, Shi G. Pentoxifylline for endometriosis. Cochrane Database Syst Rev. (2012) 1:CD007677. doi: 10.1002/14651858.CD007677

14. Hart RJ, Hickey M, Maouris P, Buckett W. Excisional surgery versus ablative surgery for ovarian endometriomata. Cochrane Database Syst Rev. (2008) CD004992. doi: 10.1002/14651858.CD004992

15. Brown J, Farquhar C. Endometriosis: an overview of cochrane reviews. Cochrane Database Syst Rev. (2014) 2014:CD009590. doi: 10.1002/14651858.CD009590

16. Brown J, Crawford TJ, Allen C, Hopewell S, Prentice A. Nonsteroidal anti-inflammatory drugs for pain in women with endometriosis. Cochrane Database Syst Rev. (2017) 1:CD004753. doi: 10.1002/14651858.CD004753

17. Abou-Setta AM, Houston B, Al-Inany HG, Farquhar C. Levonorgestrelreleasing intrauterine device (LNG-IUD) for symptomatic endometriosis following surgery. Cochrane Database Syst Rev. (2013) CD005072. doi: 10.1002/14651858.CD005072

18. Brown J, Kives S, Akhtar M. Progestagens and anti-progestagens for pain associated with endometriosis. Cochrane Database Syst Rev. (2012) 2012:CD002122. doi: 10.1002/14651858.CD002122

19. Chen I, Veth VB, Choudhry AJ, Murji A, Zakhari A, Black AY, et al. Pre- and postsurgical medical therapy for endometriosis surgery. Cochrane Database Syst Rev. (2020) 11:CD003678. doi: 10.1002/14651858.CD003678

20. Brown J, Crawford TJ, Datta S, Prentice A. Oral contraceptives for pain associated with endometriosis. Cochrane Database Syst Rev. (2018) 5:CD001019. doi: 10.1002/14651858.CD001019

21. Fu J, Song H, Zhou M, Zhu H, Wang Y, Chen H, et al. Progesterone receptor modulators for endometriosis. Cochrane Database Syst Rev. (2017) 7:CD009881. doi: 10.1002/14651858.CD009881

22. Lu D, Song H, Shi G. Anti-TNF- $\alpha$ treatment for pelvic pain associated with endometriosis. Cochrane Database Syst Rev. (2013) CD008088. doi: 10.1002/14651858.CD008088

23. Brown J, Pan A, Hart RJ. Gonadotrophin-releasing hormone analogues for pain associated with endometriosis. Cochrane Database Syst Rev. (2010) 2010:CD008475. doi: 10.1002/14651858.CD008475

24. Farquhar C, Prentice A, Singla AA, Selak V. Danazol for pelvic pain associated with endometriosis. Cochrane Database Syst Rev. (2007) CD000068. doi: 10.1002/14651858.CD000068 
25. Zhu X, Hamilton KD, McNicol ED. Acupuncture for pain in endometriosis. Cochrane Database Syst Rev. (2011) CD007864. doi: 10.1002/14651858.CD007864

26. Flower A, Liu JP, Lewith G, Little P, Li Q. Chinese herbal medicine for endometriosis. Cochrane Database Syst Rev. (2012) CD006568. doi: 10.1002/14651858.CD006568

27. Kadri HA, Hassan S, Al-Fozan HM, Hajeer A. Hormone therapy for endometriosis and surgical menopause. Cochrane Database Syst Rev. (2009) CD005997. doi: 10.1002/14651858.CD005997

28. Corte LD, Filippo CD, Gabrielli O, Reppuccia S, Rosa VLL, Ragusa R, et al. The burden of endometriosis on women's lifespan: a narrative overview on quality of life and psychosocial wellbeing. Int J Environ Res Public Health. (2020) 17:4683. doi: 10.3390/ijerph17134683

29. Bourdel N, Chauvet P, Billone V, Douridas G, Fauconnier A, Gerbaud L, et al. Systematic review of quality of life measures in patients with endometriosis. PLoS ONE. (2019) 14:e0208464. doi: 10.1371/journal.pone.0208464

30. Greil AL. Infertility and psychological distress: a critical review of the literature. Soc Sci Med. (1997) 45:1679704. doi: 10.1016/S0277-95369700102-0

31. Nussbaum M. Capabilities as fundamental entitlements: sen and social justice. Fem Econ. (2003) 9:33-59. doi: 10.1080/1354570022000077926

32. "Adaptive Preferences and Women's Options" by Martha Craven Nussbaum. Available online at: https://chicagounbound.uchicago.edu/journal_articles/ 3242/ (accessed: Oct 28, 2021).

33. Karimi M, Brazier J, Basarir H. The capability approach: a critical review of its application in health economics. Value Health. (2016) 19:7959. doi: 10.1016/j.jval.2016.05.006

34. Development as Freedom - Hardback - Amartya Sen - Oxford University Press. Available online at: https://global.oup.com/academic/product/ development/as/freedom/9780198297581 ?lang=en\&cc=no (accessed: Oct 28, 2021).

35. Anand P. Capabilities and health. J Med Ethics. (2005) 31:299303. doi: 10.1136/jme.2004.008706

36. Anand P, Hunter G, Carter I, Dowding K, Guala F, Hees MV. The development of capability indicators. J Hum Dev Capabil. (2009) 10:12552. doi: 10.1080/14649880802675366

37. Simon J, Anand P, Gray A, Rugkåsa J, Yeeles K, Burns T. Operationalising the capability approach for outcome measurement in mental health research. Soc Sci Med. (2013) 98:187-96. doi: 10.1016/j.socscimed.2013. 09.019

38. Netten A, Burge P, Malley J, Potoglou D, Towers A-M, Brazier J, et al. Outcomes of social care for adults: developing a preference weighted measure. Health Technol Asses. (2012) 16:1-166. doi: 10.3310/ hta 16160
39. The Development of a Measure of Social Care Outcome for Older People. Available online: https://www.researchgate.net/publication/254635895_The Development_of_a_Measure_of_Social_Care_Outcome_for_Older_People (accessed: Oct 28, 2021).

40. Grewal I, Lewis J, Flynn T, Brown J, Bond J, Coast J. Developing attributes for a generic quality of life measure for older people: preferences or capabilities? Soc Sci Med. (2006) 62:1891-901. doi: 10.1016/j.socscimed.2005.08.023

41. Al-Janabi H, Flynn TN, Coast J. Development of a self-report measure of capability well-being for adults: the ICECAP-A. Qual Life Res. (2012) 21:16776. doi: 10.1007/s11136-011-9927-2

42. The Capability Approach: developing an instrument for evaluating public health interventions. Available online at: https://www.gcph.co.uk/assets/0000/ 0430/Capabilities_full_report_August_08.pdf (accessed: Oct 28, 2021)

43. Forder JE, Caiels J. Measuring the outcomes of long-term care. Soc Sci Med. (2011) 73:1766-74. doi: 10.1016/j.socscimed.2011.09.023

44. Sutton EJ, Coast J. Development of a supportive care measure for economic evaluation of end-of-life care using qualitative methods. Palliat Med. (2014) 28:151-7. doi: $10.1177 / 0269216313489368$

45. Flynn TN, Chan P, Coast J, Peters TJ. Assessing quality of life among British older people using the ICEPOP CAPability (ICECAP-O) measure. Appl Health Econ Health Policy. (2011) 9:317-29. doi: 10.2165/11594150-000000000-00000

46. Comans TA, Peel NM, Gray LC, Scuffham PA. Quality of life of older frail persons receiving a post-discharge program. Health Qual Life Out. (2013) 11:58. doi: 10.1186/1477-7525-11-58

Conflict of Interest: The authors declare that the research was conducted in the absence of any commercial or financial relationships that could be construed as a potential conflict of interest.

Publisher's Note: All claims expressed in this article are solely those of the authors and do not necessarily represent those of their affiliated organizations, or those of the publisher, the editors and the reviewers. Any product that may be evaluated in this article, or claim that may be made by its manufacturer, is not guaranteed or endorsed by the publisher.

Copyright $\odot 2021$ McPherson and Bhattacharya. This is an open-access article distributed under the terms of the Creative Commons Attribution License (CC BY). The use, distribution or reproduction in other forums is permitted, provided the original author(s) and the copyright owner(s) are credited and that the original publication in this journal is cited, in accordance with accepted academic practice. No use, distribution or reproduction is permitted which does not comply with these terms. 


\section{APPENDIX}

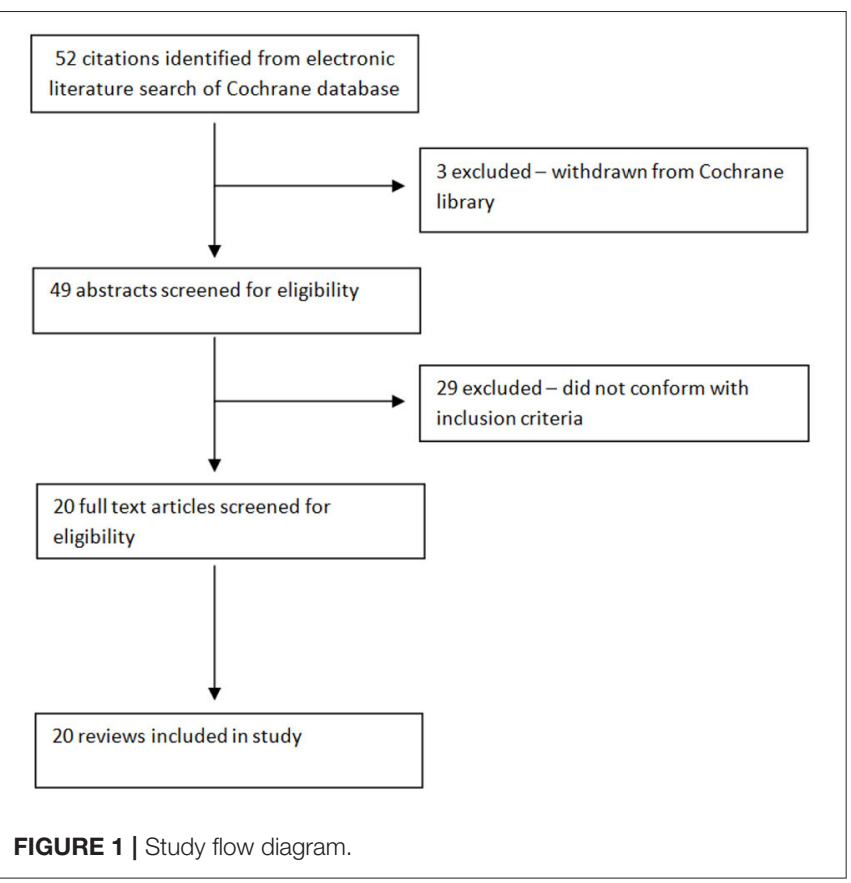

